\section{G. Burastero \\ N. Sessarego \\ G. Grappiolo \\ C. Castellazzo \\ S. Castello \\ A. Pitto \\ G. Cittadini \\ M. Podesta \\ G. Bovio \\ M. Peresi \\ E. Fulcheri \\ F. Frassoni \\ L. Spotorno}

Received: 11 January 2007

Accepted: 25 January 2007

Published online: 5 March 2007

G. Burastero (凶) • G. Grappiolo

C. Castellazzo • L. Spotorno

Hip Surgery Unit

Fondazione Scienza e Vita

Santa Corona Hospital

Via XXV Aprile 128, I-17027

Pietra Ligure (GE) Italy

N. Sessarego $\bullet$ S. Castello • A. Pitto

M. Podesta $•$ F. Frassoni

Stem Cell and Cell Therapy Center

San Martino Hospital

Genoa, Italy

G. Cittadini • G. Bovio

Department of Radiology

University of Genoa, Genoa, Italy

M. Peresi • E. Fulcheri

Pathological Anatomy Institute

University of Genoa, Genoa, Italy

\title{
Association of ex-vivo expanded human mesenchymal stem cells and rhBMP-7 is highly effective in treating critical femoral defect in rats
}

\begin{abstract}
Mesenchymal stem cells (MSC), easily culture-expanded from bone marrow, can

significantly enhance bone defect healing. Several proteins, such as the bone morphogenetic proteins (BMPs) and in particular BMP-7, are involved in bone formation in vitro and in vivo. In this preclinical study, we evaluated if the association of human MSC (hMSC) with BMP-7 had synergic action on bone healing. Rat femoral defects $(n=12)$ were treated with: autoclaved bone and mononucleated cells (MNC) as control group G1; bone and hMSC, group G2; bone with BMP-7, group G3; bone and hMSC plus BMP-7, group G4. Defect regeneration was evaluated with plain radiographs after $2,4,8$ and 12 weeks and with histological analysis. We observed organized trabeculae bridging between the osteotomic ends of the host bone in rats treated with the association of
\end{abstract}

\begin{abstract}
hMSC and rhBMP-7. These trabeculae, formed by a core of devitalized tissue surrounded by osteoblasts, osteocytes and osteoclasts, were continuous with a cortical-like structure of bony tissue. Such new bone formation of the group treated with the association of hMSC and rhBMP-7 (G4) was clearly superior compared to rats treated with rhBMP-7 (G2) or hMSC (G3) alone, as shown by radiographic analysis and histological study. The present study suggests that the association of hMSC and BMP-7 is more effective than hMSC or BMP-7 alone in the healing of femoral defects in rats. Further studies with larger samples are required to confirm these results and to evaluate the best dosage.
\end{abstract}

Keywords Bone defect $\cdot$ hMSC . rhBMP-7

\section{Introduction}

The regeneration of bone remains an elusive yet important goal in the field of orthopaedic surgery. During hip revision arthroplasty, bone-stock defects can be restored by autologous bone implantation. This procedure may lead to several complications including infection, pain, bone resorption and fracture [1]. On the other hand, the use of allogeneic bone implantation is not sufficient for massive deficit of bone stock because of its poor osteoinductive properties. In preclinical experimental models, augmentation techniques using the association of bone marrow progenitor cells and allogeneic bone or demineralized bone matrix had a synergistic effect on healing bone defects. However, biomechanical testing revealed an uncompleted structural integrity of the regenerated bone [2, 3].

More recent studies of cell-based therapies have focused on a rare population of non-hemopoietic cells, 
named mesenchymal stem cells (MSC), that is easily culture-expanded from bone marrow (BM) in an undifferentiated state. Beyond their multipotent properties on plasticity [4], BM-derived MSC are considered osteogenic precursor cells and their capacity to repair bone defects has been largely demonstrated in vivo [5-7]. Bone formation was also achieved using MSC vehiculated by carriers such as ceramics composed of hydroxyapatite/tricalciumphosphate and Ti-fibermetals, which provide a substrate for cellular attachment, proliferation and differentiation, but lack osteoinductive properties.

In an attempt to overcome this limitation, investigators began to use factor-based therapies such as bone morphogenetic proteins (BMPs). BMPs are members of the transforming growth factor beta (TGF- $\beta$ ) superfamily and most of them are involved in new bone formation in vitro and in vivo, recruiting surrounding MSCs and initiating the bone formation cascade starting with osteoblast activity [8]. In particular, recombinant human BMP-7 (rhBMP-7), has been proved efficacious in improving bone healing in orthotopic animal models and in clinical study for the treatment of nonunion of tibial fractures [9-12]. In addition, the combination of rhBMP-7 with allogeneic bone had a synergistic effect on healing large bone defects [13].

To date, no experimental study has evaluated the synergic action between hMSC and rhBMP-7 in vivo. In this study, we to treated critical bone defects with the association of human expanded MSC plus BMP-7 and autoclaved bone, to test the hypothesis that this combination can eventually lead to a potent synergic effect on bone regeneration.

\section{Materials and methods}

\section{Experimental design}

The study, approved by IST Animal Ethics Committee, was performed according to national laws on animal research, used 12 male athymic (Harlan HSD: Rh-rnu) rats (median weight, 379 g; range, 314-453 g). In all of the animals, skeletally mature, a femoral segmental defect model was produced (Fig. 1).

Rats were randomly assigned to one of four groups. In control group G1 (3 animals), the femoral gap was filled with autoclaved bone and mononucleated cells (MNC). MNC is a heterogeneous population of BM-derived cells, containing mainly haematopoietic progenitors which differentiate into all mature blood cells, but only few MSC. In group G2 (3 animals), the femoral gap was treated with autoclaved bone and hMSC; in group G3 (3 animals), the femoral gap was filled with autoclaved bone and rhBMP-7 (Stryker Biotech); and in group G4 (3 animals), the femoral gap was filled with autoclaved bone, hMSC and rhBMP-7.

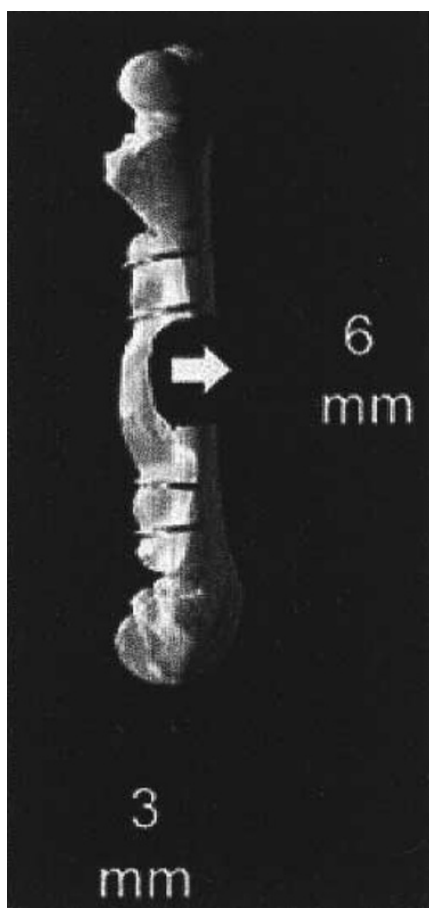

Fig. 1 Femoral segmental defect model used for the study. A 6mm gap was made at the diaphysis

Surgical method

The critical size bone defect model used in this study has already been applied to evaluate long bone repair in rats [14]. Rats were operated under general anaesthesia achieved with the intraperitoneal injection of diazepam ( $1 \mathrm{mg}$ per $100 \mathrm{~g}$ body weight $)$ and the intramuscular administration of xylazine $(0.5 \mathrm{mg}$ per $100 \mathrm{~g}$ body weight) and ketamine $\mathrm{HCl}$ (4 mg per $100 \mathrm{~g}$ body weight).

The right limb was shaved and prepared. The anterior surface of the right femur was exposed using an anterolateral approach, saving muscles and tendons. Before femoral osteotomy, a 3-mm thick and 25-mm long polymethylmethacrylate plate was fixed with four stainless steel cerclage wires. Then, a 6-mm femoral gap was realized in the central diaphysis and filled with the different graft materials.

Isolation and cultivation of human mesenchymal stem cells

BM $(50 \mathrm{ml})$ was obtained by multiple aspirations from the iliac crest of one aged male patient (75 years) undergoing hip arthroplasty. The patient gave written informed consent for his BM sample to be used. Mononuclear cells (MNC), isolated by centrifugation on Ficoll-Hypaque (Ficoll-Paque; Eurobio-Les Ulis Cedex B) were plated at a concentration of $10^{6}$ cells $/ \mathrm{ml}$ with MesenCult medium (StemCell Technologies) and incubated at $37^{\circ} \mathrm{C}$ with $5 \%$ humidified $\mathrm{CO}_{2}$ atmosphere. After $24 \mathrm{~h}$, non- 
adherent cells were discarded, fresh medium was added and replaced twice weekly. When cultures were still subconfluent, adherent cells were detached with $0.05 \%$ trypsin (Euroclone), and replated for the next generation.

\section{Preparation of implants for the different groups}

Bone filler was prepared from a $2 \%$ carboxymethyl cellulose (CMC; Sigma) hydrogel; this carrier is non-toxic and biocompatible. BMP-7, available as powder, was reconstituted with sterile saline to yield a moist paste. Then, the implants, were prepared as follows:

- G1. CMC + autoclaved bone + $60 \times 10^{6} \mathrm{MNC}$

- G2. CMC + autoclaved bone $+6 \times 10^{6} \mathrm{hMSC}$

- G3. CMC + autoclaved bone $+240 \mu \mathrm{g}$ rhBMP-7

- G4. CMC + autoclaved bone + $6 \times 10^{6} \mathrm{hMSC}+240 \mu \mathrm{g}$ rhBMP-7

Radiographic and histological evaluations

At 2, 4, 8 and 12 weeks after implantation the regeneration defect was evaluated with plain radiographs (Villa Mercury 165, 2 mAs). Two authors (GG and LS) independently and blindly evaluated the radiographs, focusing on bone density, the presence of osseous union between host bone and implant, osseous callus overlying the implant, periosteal new bone and bone graft remodelling. Bone graft was radiographically evaluated using a modified six-grade Cook classification [15] in which a seventh grade for overgrowth ossification was added, as follows: $C O$, nonvisible new bone formation; $C 1$, minimal new disorganized bone; $C 2$, disorganized new bone bridging graft to host at both ends; $C 3$, organized new bone of cortical density bridging at both ends; $C 4$, loss of graft-host distinction; $C 5$, significant new bone and graft remodelling; and C6, overgrowth ossification.

After the week-12 radiographic evaluation the animals were sacrificed by hypercapnia and the femora were cleaned of soft tissues and the fixation plates were removed. The limbs were fixed in $10 \%$ buffered formalin, subsequently dehydrated and embebbed in methylmethacrylate. The undecalcified sections were stained with toluidine blue and basic fuchsin.

\section{Results}

A femoral segmental gap model was produced in 12 athymic Rh-rnu rats, without any case of fixation failure during the course of study.

At radiographic evaluation according to the Cook classification (Table 1), control rats (group G1) scored $\mathrm{C} 0$ at all four evaluations because they showed progressive graft resorption and remodelling of the osteotomic segments without any sign of new bone formation (Fig. $2 \mathrm{a}, \mathrm{b}$ ). In group G2 (hMSC), there was minimal remodelling of the graft at two and four weeks (score, $\mathrm{C} 0-\mathrm{C} 1$ )

Table 1 Radiographic evaluation of femoral defect healing according to a modified Cook classification [15], in 12 athymic rats, by treatment group during follow-up

\begin{tabular}{|c|c|c|c|c|}
\hline Group & Week 2 & Week 4 & Week 8 & Week 12 \\
\hline \multicolumn{5}{|c|}{ G1. Control (MNC) } \\
\hline Animal 1 & $\mathrm{CO}$ & $\mathrm{C} 0$ & $\mathrm{CO}$ & $\mathrm{C} 0$ \\
\hline Animal 2 & $\mathrm{CO}$ & $\mathrm{CO}$ & $\mathrm{CO}$ & $\mathrm{CO}$ \\
\hline Animal 3 & $\mathrm{C} 0$ & $\mathrm{C} 0$ & $\mathrm{C} 0$ & $\mathrm{C} 0$ \\
\hline \multicolumn{5}{|l|}{ G2. MSC } \\
\hline Animal 4 & $\mathrm{CO}$ & $\mathrm{C} 0$ & $\mathrm{CO}$ & $\mathrm{CO}$ \\
\hline Animal 5 & $\mathrm{C} 0$ & $\mathrm{C} 1$ & $\mathrm{C} 1$ & $\mathrm{C} 2$ \\
\hline Animal 6 & $\mathrm{C} 0$ & $\mathrm{C} 1$ & $\mathrm{C} 1$ & $\mathrm{C} 1$ \\
\hline \multicolumn{5}{|l|}{ G3. BMP } \\
\hline Animal 7 & $\mathrm{C} 0$ & $\mathrm{C} 1$ & $\mathrm{C} 2$ & $\mathrm{C} 2$ \\
\hline Animal 8 & $\mathrm{CO}$ & $\mathrm{C} 1$ & $\mathrm{C} 2$ & $\mathrm{C} 3$ \\
\hline Animal 9 & $\mathrm{C} 0$ & $\mathrm{C} 1$ & NA & $\mathrm{C} 3$ \\
\hline \multicolumn{5}{|c|}{ G4. MSC + BMP } \\
\hline Animal 10 & $\mathrm{C} 0$ & $\mathrm{C} 3$ & $\mathrm{C} 4$ & $\mathrm{C} 5$ \\
\hline Animal 11 & $\mathrm{C} 1$ & $\mathrm{C} 3$ & $\mathrm{C} 5$ & $\mathrm{C} 5$ \\
\hline Animal 12 & $\mathrm{C} 1$ & $\mathrm{C} 3$ & $\mathrm{C} 4$ & $\mathrm{C} 5$ \\
\hline
\end{tabular}

$N A$, not available 

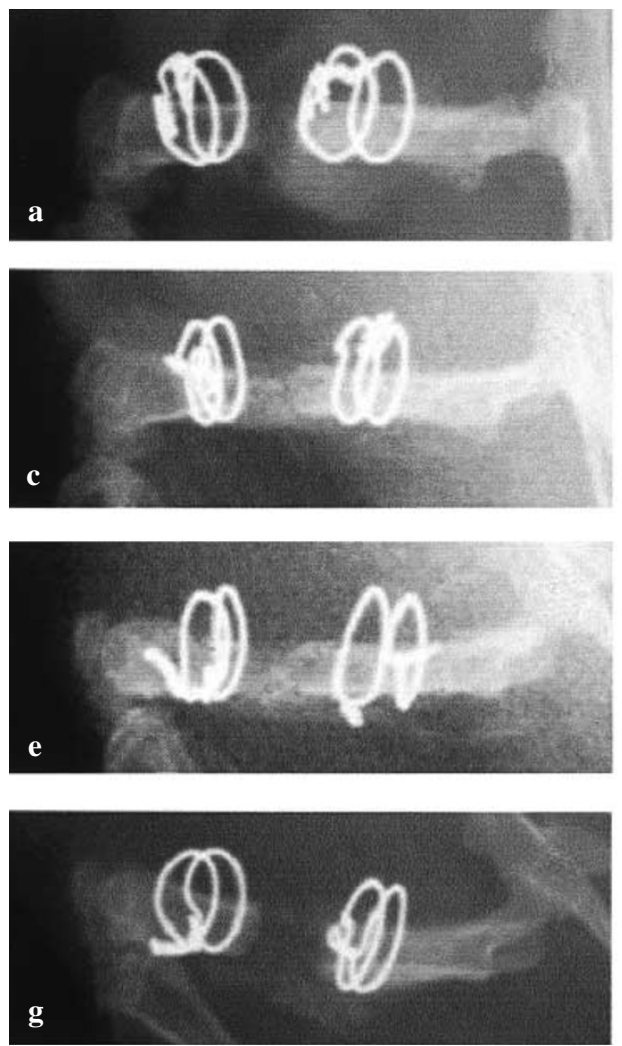
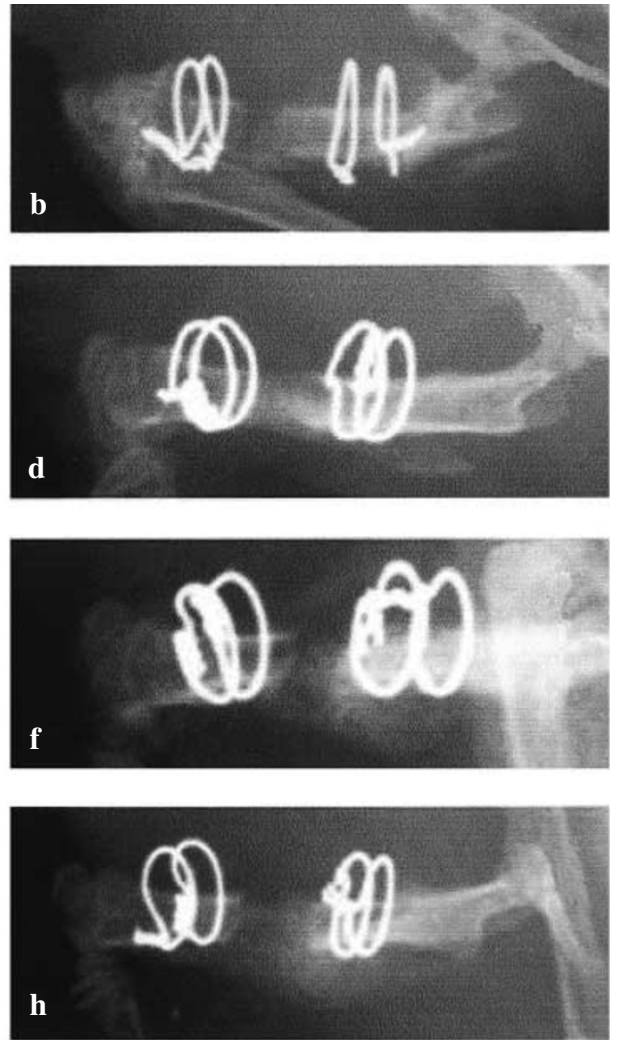

Fig. 2a-h Radiographic analysis. a, b Group G1: $60 \times 60^{6} \mathrm{MNC}$ at 2 and 12 weeks. c, d Group G2: $6 \times 60^{6}$ at 2 and 12 weeks. e, f Group G3:240 $\mu \mathrm{g}$ BMP at 2 and 12 weeks. g, h Group G4: $6 \times 60^{6}$ MSC $+240 \mu \mathrm{g} \mathrm{BMP}$ at 2 and 12 weeks
(Fig. 2c). New bone formation became evident at eight weeks, although, in all specimens, the radiographic pattern suggested that the osseous tissue was still unorganized (score, C1). At twelve weeks, we observed a bony bridge between the osteotomic ends (score, C2, animal 5) (Fig. 2d). In group G3 (rhBMP-7), osseous juxtaposition processes were present (score, $\mathrm{C} 1$ ) four weeks after surgery, as in group G2. At eight and twelve weeks, the radiographic evaluation (Fig. 2f) indicated a significant new-bone deposition (scores, C2 and C3, respectively), bridging the osteotomic ends with a well organized osseous structure. In group G4 (rhBMP-7 + hMSC), remodelling processes of the graft were observed at two weeks (score, C2) (Fig. 2g). At eight and twelve weeks, the graft connected both osteotomic ends and had a radiodensity similar to that of femoral cortex (score, C5) (Fig. 2h). Radiographic analysis documented that in groups G2, G3 and G4, the graft migrated postero-inferiorly with regard to the bony defect, as expected by gravity, because of lack of systems that provide graft restraint.

Histological examination of the bone grafts, performed at twelve weeks, confirmed the radiographic results. In group G1 (MNC), the osseous defect was filled with spread fibrous tissue, mixed with devitalized bony tissue. We did not observe bone marrow areas or viable new-bone tissue, but we found scattered inflammatory infiltrates (Fig. 3a). Group G2 (hMSC) showed new bone formation, surrounding devitalized bony tissue fragments, which appeared scattered in a widespread fibrous tissue. Some areas showed a peripheral corticallike organization, without well defined osseous bridges (Fig. 3b). In group G3 (rhBMP-7), substantial new bone formation was observed through the body of the implant and the osteotomic ends were joined by a cortical-like structure, which was in a peripherical position with respect to the graft. The samples of this group had evidence of abundant adipose tissue, inside the osseous trabeculae, with small marrow areas (Fig. 3c). In group G4 (hMSC + rhBMP-7), a complete bridging was evident between the osteotomic ends of the host bone. The new bone formation gave rise to well organized trabeculae formed by a core of devitalized tissue surrounded by osteoblasts, osteocytes and osteoclasts. The presence of such cells attested to a remarkable remodelling process. These trabeculae were continuous with a cortical-like structure of bony tissue which had considerable size and connected the ends of the graft. Moreover, every trabecula was colonized by BM (Fig. 3d). As seen already radiographically, there was a clear graft migration into a postero-inferior position and formation of a substantial amount of new bone. 

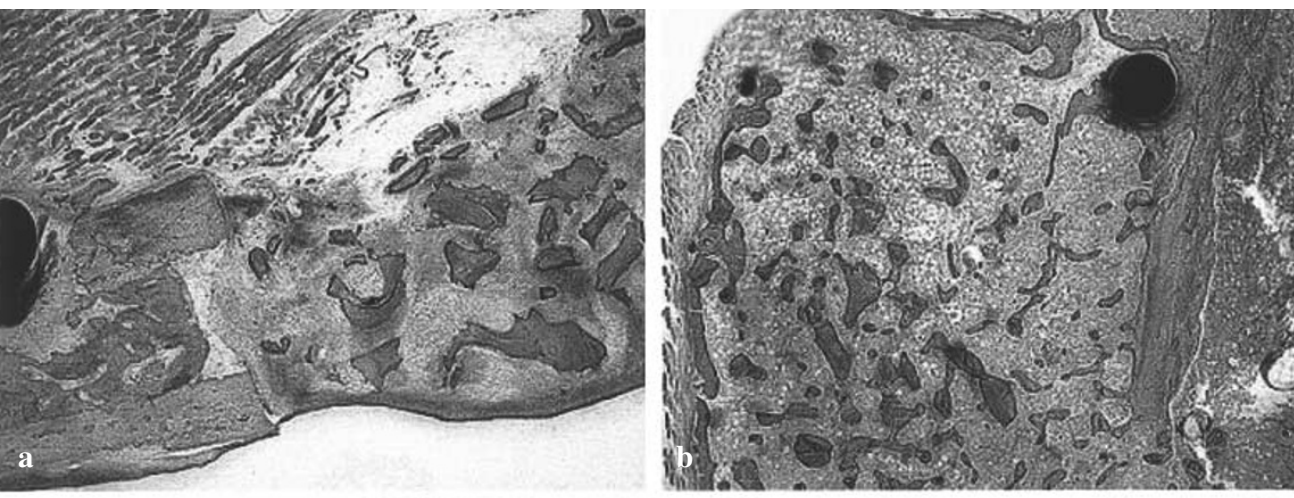

Fig. 3a-d Bone graft histological analysis performed at twelve weeks. a Group G1. b Group G2. c Group G3. d Group G4
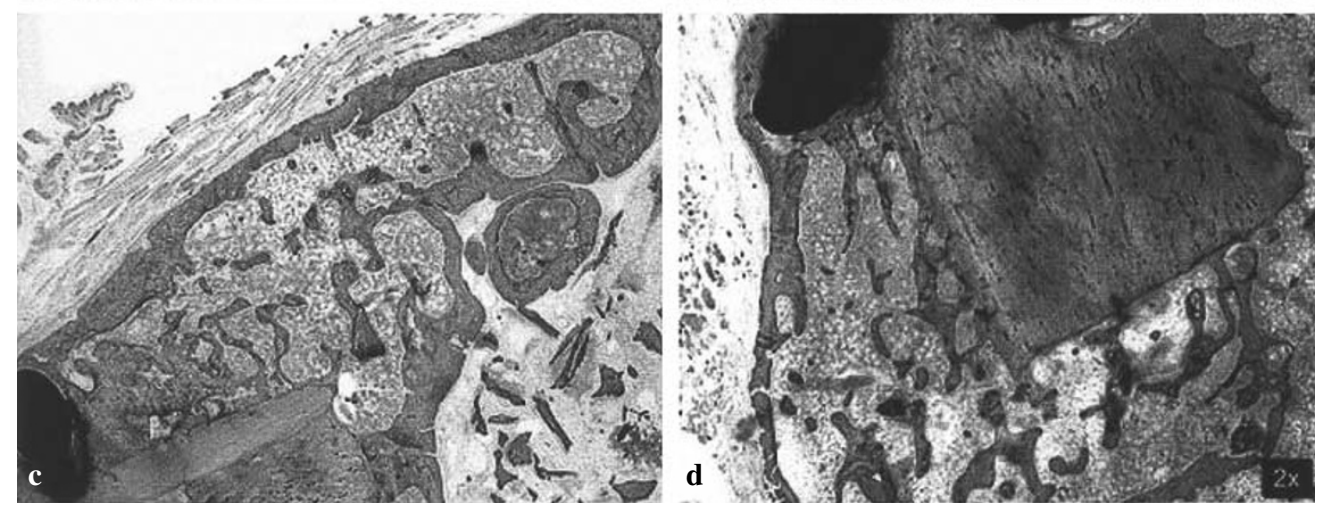

\section{Discussion}

Reconstruction of critically sized bone defects remains an important issue in orthopaedics, because the efficiency of the available therapeutic options is still limited. Recently, several experimental animal models have highlighted the potential role of cell-based therapies in order to promote repair or regeneration of skeletal tissues. In particular, BMderived MSCs have been identified as osteogenic precursors that can be induced to form bone. Moreover, hMSC are susceptible to BMPs, in particular to BMP-7, which plays a critical role in bone healing by its ability to stimulate MSC differentiation into osteochondroblastic lineage.

The aim of this study was to determine whether the combination of ex-vivo expanded hMSC and rhBMP-7 acts in a synergic fashion for bone healing of critical size defects. The results showed that in rats implanted with both expanded hMSC and rhBMP-7 (group G4), a clearly superior new bone formation was observed compared to rats treated with rhBMP-7 (group G2) or hMSC (group G3) alone. In fact, in group G4 (hMSC + rhBMP-7), radiographic analysis showed early new bone formation two and four weeks after implantation (C2 and $\mathrm{C} 3$, respectively), which improved significantly over time (C5) up to twelve weeks. Such radiographic findings were confirmed by histological studies that evidenced the formation of new bone. In the other groups, these events were not so apparent: in groups G2 (hMSC) and G3 (rhBMP-7), there was evidence of limited bone remodelling process ( $\mathrm{C} 2$ and $\mathrm{C} 3$, respectively). The lack of any new bone formation in control group G1 suggests that local rat MSC population and endogenous BMP level do not allow bone tissue repair, although associated with human MNC. On the other hand, in groups G2 and G3, the xenogenic hMSC population and rhBMP, respectively, seemed to contribute to osteogenic processes even if radiographic and histological findings suggested a more relevant impact on new bone formation by rhBMP-7 rather than hMSC.

The fact that BMP alone did not elicit bone healing in massive bone defects may be due to the low number of endogenous MSC within BM. Implanting a high number of hMSC at the site of the bone defect may be critical for two reasons:

1. hMSC differentiate into osteoblasts and produce mature bone matrix.

2. hMSC secrete inductive factors that recruit host MSC into osteogenic lineage.

Histological study of groups G3 and G4, corroborated by radiographic analysis, documented the presence of a peripheral new bone shell, rich in osteocyte lacunae. The growth of new bone of fairly good thickness, linking the osteotomic segments as a bridge, resembles the endochondral ossification which occurs during human fetal development at fourteen weeks. 
This study let us take into account minor aspects. Some investigators proved that the frequency of BM-derived hMSC decreases with age [16, 17]. However, other studies have shown that the number, proliferative and differentiation capacity of hMSC are maintained during aging $[18,19]$. In our study, it was possible to extensively expand hMSC from the $\mathrm{BM}$ of an elderly donor. Moreover, such cells retained their osteoblastic differentiation capacity.

In this work, the animal model showed a doubtless validity and the results obtained encourage us to perform new studies to verify bone formation all over the ossification process.

We cannot perform biomechanical tests, because the critical-sized femoral gap cannot heal completely without a mechanical support. Although the surgical method allows the stability of the osteotomy, the graft consolida- tion was not optimal: in fact, the absence of a scaffold determined a postero-inferior migration of the graft due to gravity. Moreover, these experiments have to be considered preliminary, because of the absence of histomorphometric analysis and the limited number of rats, which does not allow a statistical analysis. Another critical aspect is represented by the evaluation of the correct dosage of rhBMP-7 and hMSC.

In conclusion, the expanded hMSC plus rhBMP-7 association determines in vivo a significant new bone formation that may have a remarkable impact on future orthopaedic surgery strategies.

Acknowledgments This study was supported by a grant from the Italian Ministry of Health. We thank Dr. E. Ognio for assistance with the animal experiments.

\section{References}

1. Asada N, Tsuchiya H, Kitaoka K et al (1997) Massive autoclaved allografts and autografts for limb salvage surgery. A 1-8 year follow-up of 23 patients. Acta Orthop Scand 68:392-395

2. Tiedeman JJ, Connolly JF, Strates BS, Lippiello L (1991) Treatment of nonunion by percutaneous injection of bone marrow and demineralized bone matrix. An experimental study in dogs. Clin Orthop 268:294-302

3. Connolly JF, Guse R, Tiedeman J, Dehne R (1991) Autologous marrow injection as a substitute for operative grafting of tibial nonunions. Clin Orthop 266:259-270

4. Pittenger MF, Mackay AM, Beck SC et al (1999) Multilineage potential of adult human mesenchymal stem cells. Science 284:143-147

5. Deans RJ, Moseley AB (2000) Mesenchymal stem cells: biology and potential clinical uses. Exp Hematol 28:875-884

6. Bruder SP, Kraus KH, Goldberg VM, Kadiyala S (1998) The effect of implants loaded with autologous mesenchymal stem cells on the healing of canine segmental bone defects. J Bone Joint Surg Am 80:985-996

7. Bruder SP, Kurth AA, Shea $M$ et al (1998) Bone regeneration by implantation of purified, culture-expanded human mesenchymal stem cells. J Orthop Res 16:155-162
8. Urist MR (1965) Bone: formation by autoinduction. Science 150:893-899

9. Cook SD, Baffes GC, Wolfe MW et al (1994) Recombinant human bone morphogenetic protein-7 induces healing in a canine long-bone segmental defect model. Clin Orthop 301:302-312

10. Cook SD, Baffes GC, Wolfe MW et al (1994) The effect of recombinant human osteogenic protein-1 on healing of large segmental bone defects. J Bone Joint Surg Am 76:827-838

11. Cook SD, Dalton JE, Tan EH et al (1994) In vivo evaluation of recombinant human osteogenic protein (rhOP1) implants as a bone graft substitute for spinal fusions. Spine 19:1655-1663

12. Cook SD, Wolfe MW, Salkeld SL, Rueger DC (1995) Effect of recombinant human osteogenic protein-1 on healing of segmental defects in nonhuman primates. J Bone Joint Surg Am 77:734-750

13. Salkeld SL, Patron LP, Barrack RL, Cook SD (2001) The effect of osteogenic protein-1 on the healing of segmental bone defects treated with autograft or allograft bone. J Bone Joint Surg Am 83:803-816
14. Feighan JE, Davy D, Prewett AB, Stevenson S (1995) Induction of bone by a demineralized bone matrix gel: a study in a rat femoral defect model. J Orthop Res 13:881-891

15. Cook SD, Barrack RL, Santman M et al (2000) The Otto Aufranc Award. Strut allograft healing to the femur with recombinant human osteogenic protein1. Clin Orthop Relat Res 381:47-57

16. Nishida S, Endo N, Yamagiwa $H$ et al (1999) Number of osteoprogenitor cells in human bone marrow markedly decreases after skeletal maturation. J Bone Miner Metab 17:171-177

17. Majors AK, Boehm CA, Nitto $\mathrm{H}$ et al (1997) Characterization of human bone marrow stromal cells with respect to osteoblastic differentiation. J Orthop Res 15:546-557

18. Stenderup K, Justesen J, Eriksen EF et al (2001) Number and proliferative capacity of osteogenic stem cells are maintained during aging and in patients with osteoporosis. J Bone Miner Res 16:1120-1129

19. Justesen J, Stenderup K, Eriksen EF, Kassem M (2002) Maintenance of osteoblastic and adipocytic differentiation potential with age and osteoporosis in human marrow stromal cell cultures. Calcif Tissue Int 71:36-44 